\title{
Citizens' perspectives on personalized medicine: a qualitative public deliberation study
}

\author{
Yvonne Bombard ${ }^{\star, 1}$, Julia Abelson ${ }^{2}$, Dorina Simeonov ${ }^{3}$ and Francois-Pierre Gauvin ${ }^{4}$
}

Our objective was to explore citizens' informed and reasoned values and expectations of personalized medicine, a timely yet novel genomics policy issue. A qualitative, public deliberation study was undertaken using a citizens' reference panel on health technologies, established to provide input to the health technology assessment process in Ontario, Canada. The citizens' panel consisted of five women and nine men, aged 18-71 years, with one member selected from each health authority region. There were shared expectations among the citizens' panel members for the potential of personalized medicine technologies to improve care, provided they are deemed clinically valid and effective. These expectations were tempered by concerns about value for money and the possibility that access to treatment may be limited by personalized medicine tests used to stratify patients. Although they questioned the presumed technological imperative presented by personalized medicine technologies, they called for increased efforts to prepare the health-care system to effectively integrate these technologies. This study represents an early but important effort to explore public values toward personalized medicine. This study also provides evidence of the public's ability to form coherent judgments about a new policy issue. Concerned that personalized tests might be used to ration care, they suggested that treatment should be made available if patients wanted it, irrespective of tests that indicate little benefit. This issue raises clinical and policy challenges that may undermine the value of personalized medicine. Further efforts to deliberate with the public are warranted to inform effective, efficient and equitable translation of personalized medicine. European Journal of Human Genetics (2013) 21, 1197-1201; doi:10.1038/ejhg.2012.300; published online 23 January 2013

Keywords: personalized medicine; genomic testing; public engagement; deliberative methods; health policy; health technology assessment

\section{INTRODUCTION}

Personalized medicine promises to ensure that the most effective treatment is used in the most appropriate patients. The cornerstone of personalized medicine is the application of genomic biomarkers to improve the prevention, diagnosis and treatment of disease by refining individuals' estimated risks. ${ }^{1}$ Germline and somatic genomic variation is increasingly used to predict disease incidence, severity, prognosis and response to therapy in cancer care. ${ }^{1,2}$ Pharmacogenomic interventions have been used to avoid serious adverse outcomes and optimize drug dosing. ${ }^{3}$ Thus, personalized medicine has been heralded as a major transformative milestone in medicine, poised to improve health care. ${ }^{3}$

In addition to the clinical benefits, personalized medicine is touted to offer substantial health-care savings. ${ }^{4,5}$ One example is the gene expression profile (GEP) test that predicts the benefit of adjuvant chemotherapy among women with localized, estrogen receptorpositive breast cancer. ${ }^{6}$ Studies exploring GEP's clinical utility found a significant reduction in 10-year recurrence rates for women with a high recurrence score who had node-negative, receptor-positive disease, and no demonstrable reduction in distant recurrence at 10 years for the low-risk category, ${ }^{7}$ and that recurrence risks are predictive of benefit from chemotherapy. ${ }^{6}$ Despite questions of GEP's limited reliability, women with a low-risk recurrence score use GEP to learn that they are unlikely to benefit from chemotherapy and may spare themselves unnecessary treatment and exposure to toxicity, while potentially saving costs to the health-care system on the order of hundreds of millions each year. ${ }^{5}$ Commentators posit that personalized genomic medicine has the potential to 'bend the costs curve', ${ }^{4}$ presuming the investment in the technology does not offset the savings. It is thus not surprising that there is increasing interest in, and expectations for, the translation of personalized medicine tests and technologies into health care. ${ }^{8}$

However, there remain numerous challenges in the realization of personalized medicine. Alongside the need for clinical validation and utility assessment of relevant tests and technologies, there are increasing calls to engage stakeholders to support effective translation of personalized medicine. ${ }^{3,8,9}$ Stakeholder engagement is gaining increasing importance in heath technology assessment and related policy decision making, ${ }^{10-13}$ with particular calls to engage the general public. $^{10,14}$ Arguably, the public is one of the most important stakeholders in personalized medicine, yet there is a paucity of studies regarding citizens' values, concerns and expectations of personalized medicine. ${ }^{15}$ As the science, delivery and organization of personalized medicine develops, understanding the public's perceptions, values and expectations is important given that the public is not only its main beneficiary but also its primary funder.

\footnotetext{
1Yale University, School of Public Health \& Memorial Sloan-Kettering Cancer Center, Center for Health Policy and Outcomes and Clinical Genetics Service, New York, NY, USA; ${ }^{2}$ Department of Clinical Epidemiology and Biostatistics, McMaster University, Centre for Health Economics and Policy Analysis, Hamilton, Ontario, Canada; ${ }^{3}$ nstitute for Clinical Evaluative Sciences, Toronto, Ontario, Canada; ${ }^{4}$ McMaster Health Forum, McMaster University, Hamilton, Ontario, Canada

${ }^{\star}$ Correspondence: Dr Y Bombard, Yale University, School of Public Health \& Memorial Sloan-Kettering Cancer Center, Center for Health Policy and Outcomes and Clinical Genetics Service, 300 E66th Street, 14th Floor, New York, NY 10065, USA. Tel: + 1646888 4475; Fax: + 1646 227 7102; E-mail: yvonne.bombard@yale.edu

Received 19 September 2012; revised 5 December 2012; accepted 18 December 2012; published online 23 January 2013
} 
Our objective was to describe citizens' informed and reasoned values and expectations of personalized medicine - a timely genomics policy issue, on which scholars suggest that the public would have difficulty forming coherent judgments, given its relative novelty and unfamiliarity. ${ }^{16,17}$

\section{MATERIALS AND METHODS}

The Hamilton Health Sciences/McMaster University Faculty of Health Sciences Research Ethics Board approved the study, on which this article is based. Panel members gave informed consent before taking part.

\section{Citizens' reference panel on health technologies}

The citizens' reference panel on health technologies was established from 2009 to 2010 as part of a university-government research collaboration to provide input to the Ontario Health Technology Advisory Committee (OHTAC) and their Medical Advisory Secretariat (MAS) at various stages in their health technology assessment (HTA) process. ${ }^{18}$ Demographic and geographic balance was sought in creating the 14-person panel to ensure a reasonable range of perspectives on the issues being deliberated. The recruitment strategy and the panel's demographics are described in detail elsewhere. ${ }^{19,20}$ Briefly, we used a 'civic lottery system' to recruit the 14-person panel, which consisted of five women and nine men, and was meant to include one member from each local health authority region. Fifty percent of the members were Canadian, while the other $50 \%$ of participants reported to be of the following backgrounds: European, European Canadian, Asian and South Asian. Ages ranged as follows: two members between ages 18 and 24, four between 25 and 39, four between 40 and 54, two between 55 and 70 and one aged over 71 .

\section{Data collection and analysis}

The panel met on five separate occasions over 18 months to review five selected health technologies being assessed by OHTAC and MAS. Background materials were circulated in advance of each meeting including: HTA materials provided by MAS (eg, evidence summaries, draft recommendations), review articles, newspaper clippings and a workbook, which summarized the key attributes of each technology suitable for a lay audience and the discussion questions for deliberation. During each meeting, the panel engaged in informed, facilitated discussion and values elicitation through 1-day structured deliberation sessions. Each meeting began with an overview of the meeting goals (ie, deliberation about social values related to the topic) and a review of the key elements of deliberation. All panel members were encouraged to participate actively in discussions, to openly express their positions on the topics under scrutiny and the values underlying these, and to be respectful of others and their perspectives while challenging each other's ideas. Each deliberation included an overview of the background of the topic followed by a Q\&A session and a combination of large (externally facilitated by JA) and small (self-facilitated) group discussions with reporting back and thematic summarizing sessions that sought common ground while highlighting any points of divergence within the group. Collectively, these represent validated methods of deliberative processes, ${ }^{21}$ which previous studies have shown to elicit in-depth views, emphasizing informed, values-based reasoning, with the goal of reaching common ground on complex, value-laden topics. ${ }^{22-26}$ These deliberative methods are increasingly used to elicit public values on genomics policy issues. ${ }^{17,24,25,27}$

The focus of this paper is on the panel's review and deliberations of GEP for early stage breast cancer, and the general topic of personalized medicine. The panel was asked to deliberate on the ethical and societal questions raised by these technologies, based on a list of 'moral' questions developed by BjØrn Hofmann. ${ }^{28}$ They were also offered the opportunity to add questions to the assessment of these technologies, which was being conducted by OHTAC and MAS. Specifically, we asked the following three discussion questions:

1. What questions do you have about the value of GEP that you would like to see OHTAC include in its review?

2. What questions do you have about the area of personalized medicine, in general, that OHTAC could consider in its review of these new technologies?
3. Which ethical and social questions from Hofmann's list ${ }^{28}$ need to be applied to: (a) GEP; and (b) personalized medicine, in general?

To prepare the panel for the deliberation on personalized medicine, we defined the term and described the potential benefits and current challenges, including (but not exclusive to): informed treatment or diagnosis, reduced side effects, reduced health-care costs, and the challenges of provider expertise and laboratory capacity, the potential for insurance and employment discrimination based on genetic status, as well as the potential costs associated with conducting the tests and ensuring test quality and confidentiality. To prepare the panel for the deliberation on GEP, we described how the test works and how to interpret the recurrence scores. We also described the potential benefits of informing treatment decisions by learning more information about a patient's recurrence risk and benefit of chemotherapy, as well as the limitations of the test's effectiveness and reliability.

Discussions were recorded and transcribed verbatim. Transcripts of meeting discussions were analyzed using content analysis, incorporating principles of constant comparison ${ }^{29}$ and qualitative description ${ }^{30,31}$ by YB. Codes pertaining to the perceptions of GEP and personalized medicine were analyzed to identify the main expectations and concerns in their use and integration into health care. Thematic arguments were identified and then fully described to capture underpinning values or judgments as discussed by the study team. Emerging reasoning was then contrasted with existing data, and summarized below as the main themes arising from the panel's deliberations.

\section{RESULTS}

There were shared expectations among the citizens' panel members for the potential for personalized medicine technologies to improve care, provided they are deemed clinically valid and effective. These expectations were tempered by concerns about costs, access, need and feasibility of adoption into the health system, summarized as themes below using illustrative quotes from the deliberations.

\section{Considering costs and opportunity costs}

The costs of developing and integrating personalized medicine technologies were especially germane for the panel. For some, costs were inherent to the value of these technologies. Members questioned whether the value for money gained from personalized medicine may be influenced by the interests of the producers of the technologies:

Nancy (Aliases have been used to protect participants' anonymity.):... What are the interests of the producers of technology? What are the interests of the users of the technology? But to me that should be a twofold question. Do they coincide or ... are we going to get what we think we're getting from this expensive investment?

The opportunity costs of allocating resources to support the integration of personalized medicine were also emphasized during the deliberations. Panel members traded off those costs with the opportunity costs of not funding other resources or interventions. In reporting back from his small group discussion, one member referred to the opportunity costs of funding the GEP test versus interventions to reduce the incidence of death from breast cancer:

Frank: We talked about the cost of implementing it versus the people that we are losing to cancer in terms of the women that are dying of breast cancer. We talked about you know I think it was, was it $4 \%$ of cancer, breast cancer patients that are dying from it? Yea. So we're just doing a balancing there of you know the cost of implementing it (GEP test) versus the value of it to the families that are facing breast cancer.

However, discussions about costs were complicated by panel members' concern that personalized medicine tests and technologies might influence patients' access to care. 


\section{Tailoring care or limiting access to care?}

Members raised the possibility that the results of personalized medicine tests may limit patients' access to treatment. Members discussed GEP tests and patients who fall into the low recurrence risk category, in particular. They suggested that the results of the GEP test should not preclude a patient from undergoing chemotherapy if she wants it. For them, there should be access to treatment despite the low likelihood of benefit:

Kyle: I was saying basically for instance if you reach a genetic expression profiling and you were in that number of... low risk that you still could yourself make a decision: 'well you know what? I still want to go in and get the chemotherapy'. But maybe because of a cost issue they're saying: 'well no you're not in that category so you're not able to get this'.

Members believed that treatment decisions should ultimately be the patients' choice, because treatment might offer hope:

Benjamin: I was thinking that if there was a chance of moving forward with chemotherapy now without doing gene testing and that chemotherapy was going to offer some advantage to the whole treatment process, then I felt strongly that the individual should be able to go make that decision at that point (to) go ahead with it (chemo). Where(as) if they had the gene testing and it proved that they fall short of the group that was really going to benefit from it, might they be taking away that small little help, that little bit of help, that they might need to overcome what they're facing right now?

Some went as far to suggest that patients' preferences for following the course of action recommended by personalized genomic tests be explored before ordering them to maximize their utility and reduce unnecessary costs. Resource allocation decisions were important for those that challenged the presumed technological imperative to embrace personalized medicine.

\section{Questioning the technological imperative}

Members were skeptical of the promises and need for personalized medicine. Some questioned the imperative - or 'obligation' - that the availability of these tests engender, raising concerns that it 'preys' on patients' emotional vulnerabilities:

Nancy: I think there's this whole emotional thing that's preying upon people's decision making too and the people who develop these technologies aren't they throwing that (emotional vulnerability) at you? ... it's not just developing the technology so it suits Canadians for Canadians but it's are we feeling obligated to buy into it (technology) cause it's out there? I think that's a very powerful thing that has to be investigated.

Some suggested that rational adjudication of the technology might not be possible for vulnerable groups who may have few options or might otherwise feel compelled to avail themselves of the new technology. Despite these criticisms, some felt an obligation to consider personalized genomic technologies in hopes that they might improve care, especially for patients with limited options:

Larry: What's the option though? I mean if we don't go ahead with this genetic testing and leave the status quo then things are certainly not going to improve.

In order to 'improve' care, members focused on the need to prepare the health-care system to effectively integrate personalized medicine.

\section{Preparing the health-care system}

Panel members uniformly believed that the health-care system was generally unprepared for the adoption of personalized medicine. They called for increased public awareness to increase confidence and use of the technology, in addition to counseling services for those facing new choices and decisions concerning these technologies.

The need to prepare the health-care system also extended to strengthening laboratory quality assurance mechanisms and training of providers to ensure they can effectively interpret the results of these personalized genomic tests. Members reasoned that increasing provider knowledge was important to prevent a loss of credibility:

Paula: I think there's also that duty to be able to advise frontline practitioners whether it's a health care nurse or whether it's the family doctor ... (those) key point persons that have therapeutic relationships with patients that (can) have access to as much information. If they don't have the information, (then) at least knowing where the resources are to be able to find out that information. That's a crucial piece because otherwise we just totally lose the credibility.

The general feeling of unpreparedness led some to question whether it was premature to adopt personalized medicine. Some spoke about a sense of 'courage' that the Ministries of Health need to possibly reconsider the diffusion of these technologies:

Frank: We questioned the whole timing of the release and the courage for OHTAC and the Ministry to also step back and say: 'perhaps now is not the timing'... In general, we talked about what it will take to bring the health system up to speed or up to par in order for that release to actually happen.

\section{DISCUSSION}

There were shared expectations among the citizens' panel members for the potential of personalized medicine technologies to improve care, provided they are deemed clinically valid and effective. These expectations were tempered by concerns about value for money and the possibility that access to treatment may be limited by results of tests used to stratify patients. Although they questioned the presumed technological imperative to incorporate personalized medicine technologies into health care, they called for increased efforts to prepare the health-care system to effectively integrate these technologies.

One of the novel findings was the conviction that access to treatment regimes should not be determined by the results of personalized genomic tests. The citizens' panel asserted that treatment should be made available if patients wanted it, irrespective of tests that indicate little to no benefit. Their point was that faced with the possibility of a recurring cancer, patients may opt to avail themselves of any opportunity they can, regardless of the potential for harm. This was rooted in a concern that personalized genomic tests might be used to ration care. Indeed, by virtue of personalized medicine's ability to stratify patients who will likely benefit from treatment from those who will not, patients who fall into the latter category may find themselves, effectively 'orphaned', without treatment options or possibly without coverage for insured treatments. This issue raises important challenges for providers who may be faced with a growing pool of patients demanding access to treatments that may not benefit them. This issue also highlights broader ethical challenges in balancing autonomy with resource allocation. It is widely accepted in clinical ethics that at some point in a person's life there will be no 
further suitable options, where it may not be ethically defensible to pursue futile treatments, even if a person would like certain things to be done. ${ }^{32,33}$ Given the possibility of an increasing pool of patients that may find themselves without treatments options from personalized medicine tests, it might be useful to explore alternative treatments options, so that people are not left without hope nor request futile treatments.

Further, providing treatment that is unlikely to benefit patients undermines the clinical utility of these tests since their results are not being used to inform treatment decision making, and by extension, undermine their value for money. The citizens' panel appreciated this tension and suggested that patient preferences for following the course of action recommended by personalized genomic tests be explored before ordering them, to maximize their utility and value for money. Although, on the one hand, this proposal may represent a way forward, on the other, restricting the use of tests based on patients' initial inclinations to adhere to test results undermines the general utility of personalized medicine, should majorities of patients decline to use relevant tests. These issues highlight the general tension between descriptive ethics (ie, what people believe) and normative ethics (ie, what ethical principles oblige); that is, what the public thinks should not necessarily determine what we ought to do. Using public engagement to explore values can identify issues, clarify meaning and broaden perspectives, ${ }^{34}$ toward enhancing the decisions made. However, such values elicitation exercises cannot and should not - necessarily determine policy decisions. Just as efficacy or cost-effectiveness evidence cannot independently determine policy, values evidence derived from public engagement cannot alone determine the policy decision. ${ }^{19}$ Nonetheless, these perspectives provide timely insight into the complexity of the issues posed by the introduction of personalized medicine technologies, which could inform the equitable translation of personalized medicine into the health-care system.

More broadly, restricting access to treatment based on the results of genomic tests mirrors growing concerns about genetic discrimination internationally. ${ }^{35}$ Arguably, issues of genetic discrimination play out quite differently for families with hereditary diseases than those in the general population using personalized genomic tests. Instead of concerns that insurers will use genetic information to impose high premiums and deny coverage or employment opportunities, as in the case of hereditary diseases, ${ }^{36}$ patients may perceive themselves to be treated differently in their access to various treatments. ${ }^{37}$ Indeed, as larger segments of the population are treated with personalized medicine tests and technologies, a different form of genetic discrimination may be introduced. Policy and practice interventions are warranted to mitigate this issue from becoming an unnecessary barrier to the realization of personalized medicine. Unfortunately, the classic form of genetic discrimination still persists unabated in some parts of the world, including Canada - the only G8 country without relevant policy protections. ${ }^{38}$

There is also a growing need to improve public and professional knowledge of genomics and, now, personalized medicine. ${ }^{39-43}$ Although these educational needs certainly existed before the emergence of personalized medicine, it is hoped that the increasing interest in the translation of personalized medicine tests and technologies will renew efforts to increase public and professional discourse of the benefits and risks of personalized genomic tests that aim to stratify patients and inform treatment decision making, and not ration health care.

This study represents an early but important effort to explore public values toward personalized medicine in one jurisdiction in
Canada. This study also provides evidence of the public's ability to form coherent judgments and expectations about a new or future policy issue using structured, facilitated deliberative discussion that took place among a small group of citizens over several hours using recognized methods. ${ }^{22-25}$ Although not intended to represent the views of the general population, the findings contribute novel and timely insights into the perceptions, values and concerns that a group of citizens and potential users hold toward personalized medicine. Importantly, the citizens' panel was concerned that personalized genomic tests might be used to ration care. This issue raises significant clinical and policy challenges and has the potential to undermine the clinical utility and value of personalized genomic tests. Additional studies of this kind, using well-designed methods of public deliberation with more demographically diverse members on a range of personalized medicine technologies in other jurisdictions are warranted to inform the effective, efficient and equitable translation of personalized medicine into the health-care system.

\section{CONFLICT OF INTEREST}

The authors declare no conflict of interest.

\section{ACKNOWLEDGEMENTS}

We are grateful to our citizens' panel members for generously sharing their time and insights with us over the course of this 18-month research study. The project was led by Dr Abelson at McMaster University with funding generously provided by the Ontario Ministry of Health and Long-Term Care through the Ontario Health Technology Evaluation Fund. Dr Bombard is supported by a CIHR Postdoctoral Fellowship, and was supported by the CIHR Strategic Training Fellowships of 'Public Health Policy' and 'Health Care, Technology and Place' at the time of this study. DS was supported by the Ontario Graduate Scholarship at the time of this study. We thank Lyn Sauberli for administrative assistance provided throughout this project and the Center for Health Economics and Policy Analysis at McMaster University for infrastructure support. We also thank Mark Schlesinger for insightful discussions on this article, and the anonymous reviewers for their constructive comments.

1 McDermott U, Downing JR, Stratton MR: Genomics and the continuum of cancer care N Engl J Med 2011; 364: 340-350.

2 Weitzel JN, Blazer KR, Macdonald DJ, Culver JO, Offit K: Genetics, genomics, and cancer risk assessment: state of the art and future directions in the era of personalized medicine. CA Cancer J Clin 2011: 61: 327-359.

3 Hudson TJ: Personalized medicine: a transformative approach is needed. CMAJ 2009, 180: 911-913.

4 Armstrong K: Can genomics bend the cost curve? JAMA 2012; 307: 1031-1032.

5 Lyman GH, Cosler LE, Kuderer NM, Hornberger J: Impact of a 21-gene RT-PCR assay on treatment decisions in early-stage breast cancer: an economic analysis based on prognostic and predictive validation studies. Cancer 2007; 109: 1011-1018.

6 Paik S, Tang G, Shak S et al: Gene expression and benefit of chemotherapy in women with node-negative, estrogen receptor-positive breast cancer. J Clin Oncol 2006; 24: 3726-3734.

7 Paik S, Shak S, Tang G et al: A multigene assay to predict recurrence of tamoxifentreated, node-negative breast cancer. N Engl J Med 2004; 351: 2817-2826.

8 Doerr M, Eng C: Personalised care and the genome. BMJ 2012; 344: e3174.

9 Auffray C, Caulfield T, Khoury MJ, Lupski JR, Schwab M, Veenstra T: Genome Medicine: past, present and future. Genome Med 2011; 3: 6

10 Head S: NICE should engage generalists-public and professional. BMJ 2009; 338 b639.

11 Rawlins MD, Culyer AJ: National Institute for clinical excellence and its value judgments. BMJ 2004; 329: 224-227.

12 Davies C, Wetherell M, Barnett E, Seymour-Smith S: Opening the box: Evaluating the Citizens Council of NICE 2005 vol Report prepared for the National Coordinating Centre for Research Methodology, NHS Research and Development Programme.

13 Abelson J, Giacomini M, Lehoux P, Gauvin FP: Bringing 'the public' into health technology assessment and coverage policy decisions: from principles to practice. Health Policy 2007; 82: 37-50.

14 Chafe R, Levinson W, Hebert PC: The need for public engagement in choosing health priorities. CMAJ 2010; 183: 165 
15 Moldrup C, Nielsen LF: Lay perspectives on pharmacogenomics: a literature review. Pers Med 2006; 3: 311-316.

16 Schlesinger M: Lumping and splitting: understanding public attitudes regarding health policy; in: Morone J, Ehlke D (eds) Health Politics and Policy. Lifton Park, New York: Delmar Cenage, 2013.

17 MacKenzie M, O'Doherty K: Deliberating future issues: minipublics and Salmon genomics. J Public Deliberation 2011; 7

18 Johnson AP, Sikich NJ, Evans G et al: Health technology assessment: a comprehensive framework for evidence-based recommendations in Ontario. Int J Technol Assess Health Care 2009; 25: 141-150.

19 Bombard Y, Abelson J, Simeonov D, Gauvin FP: Eliciting social values and ethics in health technology assessment: a participatory approach. Soc Sci Med 2011; 73: 135-144.

20 Abelson J, Bombard Y, Gauvin FP, Simeonov D: Consulting with Ontario citizens about health technologies: final report of the citizens' reference panel on health technologies; in: Ontario of Health and Long-Term Care (ed) Toronto, Ontario: Ontario Ministry of Health and Long-Term Care, 2010.

21 Abelson J, Forest PG, Eyles J, Casebeer A, Martin E, Mackean G: Examining the role of context in the implementation of a deliberative public participation experiment: results from a Canadian comparative study. Soc Sci Med 2007; 64: 2115-2128.

22 Blacksher E, Diebel A, Forest PG, Goold SD, Abelson J: What is public deliberation? Hastings Cent Rep 2012; 42: 14-17.

23 Goold SD, Neblo MA, Kim SY, Vries RD, Rowe G, Muhlberger P: What is good public deliberation? Hastings Cent Rep 2012; 42: 24-26.

24 Molster $C$, Maxwell S, Youngs $L$ et al: Blueprint for a deliberative public forum on biobanking policy: were theoretical principles achievable in practice? Health Expect 2011; PMID:21645188.

25 O'Doherty KC, Hawkins A: Structuring public engagement for effective input in policy development on human tissue biobanking. Public Health Genomics 2012; 13: 197-206.

26 O'Doherty K, Gauvin FP, Grogan C, Friedman W: Implementing a public deliberative forum. Hastings Cent Rep 2012; 42: 20-23.

27 Bombard Y, Miller FA, Hayeems RZ et al: Citizens' values regarding research with stored samples from newborn screening in Canada. Pediatrics 2012; 129: 239-247.

28 Hofmann B: Toward a procedure for integrating moral issues in health technology assessment. Int J Technol Assess Health Care 2005; 21: 312-318.
29 Strauss ACJ: Basics of Qualitative Research: Techniques and Procedures for Developing Grounded Theory, Second Edition Thousand Oaks: Sage Publications, 1998.

30 Sandelowski M: Whatever happened to qualitative description? Res Nurs Health 2000; 23: $334-340$.

31 Sandelowski M: What's in a name? Qualitative description revisited. Res Nurs Health 2010; 33: 77-84.

32 Lawrence S, Willmott L, Milligan E, Winch S, White B, Parker M: Autonomy versus futility? Barriers to good clinical practice in end-of-life care: a Queensland case. Med J Aust, 196: 404-405.

33 Stewart CL: A defence of the requirement to seek consent to withhold and withdraw futile treatments. Med J Aust, 196: 406-408.

34 Kenny N, Giacomini M: Wanted: a new ethics field for health policy analysis. Health Care Anal 2005; 13: 247-260.

35 Otlowski M, Taylor S, Bombard Y: Genetic discrimination: international perspectives. Annu Rev Genomics Hum Genet 2012; 13: 433-454.

36 Bombard Y, Veenstra G, Friedman JM et al: Perceptions of genetic discrimination among people at risk for Huntington's disease: a cross sectional survey. BMJ 2009; 338: b2175.

37 McClellan KA, Avard D, Simard J, Knoppers BM: Personalized medicine and access to health care: potential for inequitable access? Eur J Hum Genet 2012; e-pub ahead of print 11 July 2012; doi:10.1038/ejhg.2012.149.

38 Bombard Y, Palin J, Friedman JM et al: Factors associated with experiences of genetic discrimination among individuals at risk for Huntington disease. Am J Med Genet B Neuropsychiatr Genet 2011; 156B: 19-27.

39 Williams MS: The public health genomics translation gap: what we don't have and why it matters. Public Health Genomics 2012; 15: 132-138.

40 Molster C, Charles T, Samanek A, O'Leary P: Australian study on public knowledge of human genetics and health. Public Health Genomics 2009; 12: 84-91.

41 Hahn S, Letvak S, Powell K et al: A community's awareness and perceptions of genomic medicine. Public Health Genomics 2010; 13: 63-71.

42 Carroll JC, Rideout AL, Wilson BJ et al: Genetic education for primary care providers: improving attitudes, knowledge, and confidence. Can Fam Physician 2009; 55: e92-e99.

43 Skirton H, Lewis C, Kent A, Coviello DA: Genetic education and the challenge of genomic medicine: development of core competences to support preparation of health professionals in Europe. Eur J Hum Genet 2010; 18: 972-977. 